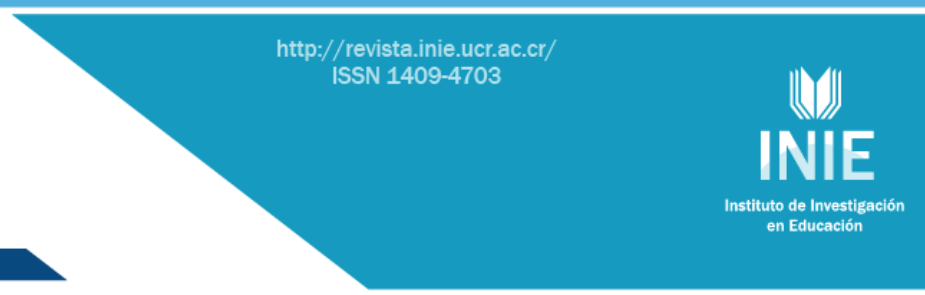

\title{
ENFOQUE TRANSVERSAL Y LONGITUDINAL EN EL ESTUDIO DE PATRONES DE APRENDIZAJE EN ALUMNOS UNIVERSITARIOS DE INGENIERIA
}

CROSS AND LONGITUDINAL APPROACH IN THE STUDY LEARNING PATTERNS OF ENGINEERING UNIVERSITY STUDENTS

\section{Volumen 16, Número 3 \\ Setiembre-Diciembre}

pp. $1-20$

\section{Este número se publicó el $1^{\circ}$ de setiembre de 2016}

DOI: http://dx.doi.org/10.15517/aie.v16i3.26089

Mariela Lourdes González

Hilda Emilia Difabio de Anglat

Revista indizada en REDALYC, $\underline{\text { SCIELO }}$

Revista distribuida en las bases de datos:

LATINDEX, DOAJ, E-REVIST@S, IRESIE, CLASE, DIALNET, SHERPA/ROMEO, QUALIS,

Revista registrada en los directorios:

ULRICH'S, REDIE, RINACE, OEI, MAESTROTECA, PREAL, CLACSO 


\title{
ENFOQUE TRANSVERSAL Y LONGITUDINAL EN EL ESTUDIO DE PATRONES DE APRENDIZAJE EN ALUMNOS UNIVERSITARIOS DE INGENIERIA

\author{
CROSS AND LONGITUDINAL APPROACH IN THE STUDY LEARNING PATTERNS OF \\ ENGINEERING UNIVERSITY STUDENTS
}

\author{
Mariela Lourdes González 1 \\ Hilda Emilia Difabio de Anglat ${ }^{2}$
}

Resumen: En el presente artículo se analiza el modelo de Vermunt en el contexto argentino, a partir de muestras procedentes de dos facultades de Ingeniería con la finalidad de disponer de una base teórica y contextual que nos permita optimizar los procesos de enseñanza y aprendizaje en la universidad. Se implementan dos estudios uno transversal y el otro longitudinal-; en el primero, se comparan estudiantes de Ingeniería del tramo inicial -1ำ año- $(N=149)$ con el final $-5^{\circ}$ año- $(N=90)$; en el segundo, se confrontan los resultados en estudiantes de primer año de Ingeniería en Ciencias Agrarias: al comienzo $(N=110)$ y al final $(N=98)$. El instrumento utilizado fue el Inventory of Learning Styles, en la versión en español de 2009, que 'permite la conformación de cuatro patrones: el orientado al significado, el orientado a la aplicación, el reproductivo y el no orientado. En el primer estudio, se efectúa un análisis jerárquico de conglomerados para identificar los patrones de aprendizaje que se conforman, En el segundo, se analizan los cambios intraindividuales en las medias de las escalas del ILS. En ambos estudios se concluye que los resultados evidencian que los patrones que se conforman con claridad son el orientado al significado y a la reproducción.

Palabras clave: ESTUDIANTES UNIVERSITARIOS, INGENIERÍA, ESTUDIO TRANSVERSAL, ESTUDIO LONGITUDINAL, PATRONES DE APRENDIZAJE, MODELO DE VERMUNT

\begin{abstract}
In the present article analyzes the model itself Vermunt in the Argentine context, one from samples from two faculties of Engineering with the aim of alienating a base of theoretical and contextual let us allow the teaching optimize and learning at the university. Two cross-one studies and other longitudinally are implemented; in the first, if you compare Engineering Students -1st initial segment ANOVA $(N=149)$ with the end of -5 ANOVA (N = 90); in the second, the results are compared in primer year students in Agricultural Engineering Sciences: the beginning $(N=110)$ and end $(N=98)$. The instrument used was the Learning Styles Inventory, in the spanish version 2009, which permit the formation of four patterns: the meaning oriented, application oriented, reproductive and undirected. In the first study a hierarchical cluster analysis to identify patterns of learning that takes place up. In the second, the intra-Individual changes are discussed in the middle of ILS scales. In both studies concluded, the results show that the patterns that make the child clearly oriented towards the meaning and reproduction.
\end{abstract}

Key words: UNIVERSITY STUDENTS, ENGINEERING, CROSS-SECTIONAL STUDY, LONGITUDINAL STUDY, PATTERNS OF LEARNING, VERMUNT'S MODEL.

\footnotetext{
1 Catedrática en la Universidad Nacional de Cuyo. Mendoza, Argentina. Becaria Posdoctoral del Consejo Nacional de Investigaciones Científicas y Técnicas, Centro de Investigaciones Cuyo, Mendoza, Argentina. Profesor investigador de la Universidad Panamericana, Campus Guadalajara, México. Doctora en Ciencias de la Educación. Dirección electrónica: marielalourdesgonzalez@yahoo.com.ar

2 Catedrática en la Universidad Nacional de Cuyo. Mendoza, Argentina. Investigadora independiente del Consejo Nacional de Investigaciones Científicas y Técnicas, Centro de Investigaciones Cuyo, Mendoza, Argentina. Doctora en Ciencias de la Educación. Dirección electrónica:ganglat@gmail.com
}

Artículo recibido: 14 de octubre, 2015

Enviado a corrección: 6 de abril, 2016

Aprobado: 11 de julio, 2016 


\section{Introducción}

La educación superior espera que los alumnos avanzados demuestren niveles profundos de procesamiento, pensamiento crítico y dominio de estrategias de regulación, en comparación con los novatos en quienes se admiten estrategias más reproductivas. Según González (2015) esta variación del énfasis de las demandas en este nivel se va a reflejar en un incremento de la orientación al significado.

En este trabajo se trata de analizar cómo progresan los patrones de aprendizaje a medida que los alumnos avanzan en sus estudios universitarios, esto es, determinar si las estrategias de aprendizaje y de autorregulación, la concepción del conocimiento, los factores motivacionales -todos ellos componentes de los patrones de aprendizaje-, conforman un estilo personal de aprendizaje progresivamente más coherente, a la vez que flexible y adaptable a los requerimientos académicos de las trayectorias estudiantiles. Es decir si los estudiantes manifiestan rasgos de patrones orientados al significado, en el cual desarrollan el pensamiento crítico hacia una perspectiva personal. Vermetten, Vermunt y Lodewijks (1999) abordan esta cuestión y mencionan que la estructura difusa que poseen los alumnos respecto de las estrategias empleadas en el aprendizaje durante los dos primeros años, se debe a un período de adaptación a un nuevo entorno de aprendizaje, que denominan período de fricción, el cual si no se supera puede conducir a la deserción tan frecuente en la trayectoria inicial del nivel universitario. Por ello, es importante indagar en alumnos argentinos si se verifican estos cambios a través del tiempo en las estrategias empleadas y en los componentes del aprendizaje.

Durante años, la investigación de cómo aprende el estudiante ha evolucionado en muchas direcciones. Una parte importante de los estudios se ha centrado en los aspectos cognitivos del aprendizaje (Kolb, 1984; Sadler-Smith, 1996), las concepciones sobre el aprendizaje o sobre algunas estrategias específicas (Marton y Säljö, 1976; Säljö, 1979), aspectos de la autorregulación (Pintrich y García, 1993; Zimmerman, 2000), de la metacognición (Bernard, 1993; Difabio de Anglat, 1999; Flavell, 1987; Martínez-Fernández, Villegas y Martínez Torres, 2004; Wittrock, 1991) y aspectos motivacionales (Alonso Tapia, 1997; Biggs, 1987, Nuttin, 1980,1982).

Un aspecto compartido por muchos de estos trabajos es la búsqueda de las vinculaciones entre las áreas de interés y el intento de arribar a constructos de estilos (García Retana, 2013) o enfoques de aprendizaje (Jonassen y Grabowoski, 1993). En Holanda, Vermunt (1992) ponderó la viabilidad de integrar un número importante de 
investigaciones previas e incorporó varias de ellas en un modelo que se centra en la relación entre estrategias de aprendizaje (cognitivas y regulatorias), concepciones de aprendizaje y orientaciones respecto del mismo (aspectos motivacionales).

La investigación de los estilos, enfoques o patrones de aprendizaje -denominaciones en uso que responden a distintos encuadres teóricos- constituye hoy un corpus extendidamente desarrollado con varios modelos e instrumentos (cfr., entre muchos otros, Aguilar Rivera, 2010; Entwistle, McCune y Walker, 2001; García-Fuentes, Muñoz Cantero y Abalde Paz, 2002; Marín Gracia, 2002). El término estilo, empleado en un principio por el autor del modelo que se sigue en este trabajo, Jan Vermunt, podía dar lugar a que se lo entendiera como un atributo invariante, un rasgo de personalidad; por esa razón, lo reemplazó por el de pattern, esto es, patrón (Vermunt, 1996, 1998, 2005), que alude a una forma de estudiar y de considerar la actividad de aprendizaje relativamente estable pero no inmutable.

En efecto, según Vermunt (1998), un estilo de aprendizaje se caracteriza por un ensamble interrelacionado de componentes del aprendizaje, tales como las actividades que habitualmente desarrolla el alumno, cómo regula los procesos, cuáles son sus concepciones y motivos para estudiar. Vermunt y Minnaert (2003) señalan que el término estilos refiere a un aprendizaje más habitual o a un tipo de rasgo. A fin de tomar en consideración la modificabilidad del constructo, proponen el uso de un término más neutral, "patrones de aprendizaje" (cfr., también, Vermunt, 2005).

\section{Modelo de Vermunt}

Vermunt (1996, 1998, 2005) conceptualiza el patrón de aprendizaje como un constructo que alude a la forma de estudiar y de considerar la actividad de aprendizaje relativamente estable pero no inmutable. Refiere a un interjuego entre un conjunto de factores relativos al aprendizaje que están afectados por ciertas variables personales y contextuales. Esto supone que el término "patrones de aprendizaje" lleva implícita una concepción de posibilidad de desarrollo a través del tiempo en función de dichos factores, rasgo que no se asume en la concepción de los estilos de aprendizaje, más estables e invariantes.

El modelo incluye cuatro componentes: estrategias de procesamiento, de regulación, concepciones de aprendizaje (modelos mentales) y orientaciones motivacionales. Las primeras refieren a las actividades intelectuales que el alumno emplea para procesar la 
información a fin de obtener ciertos resultados del aprendizaje (como, por ejemplo, aprehender los núcleos temáticos de un material de estudio). Las estrategias de regulación son aquellas actividades que el estudiante aplica para planificar, monitorear y controlar las estrategias y los propios procesos cognoscitivos. Las concepciones de aprendizaje remiten a las redes de supuestos y conceptos acerca del conocimiento y el aprendizaje. Las orientaciones motivacionales son las metas personales, intenciones, expectativas, dudas, etc., que un alumno puede experimentar durante el trayecto formativo.

Sobre la base de una extensa investigación empírica, mediante el análisis de componentes principales, Vermunt (1998) identifica cuatro patrones: dirigido al significado, dirigido a la reproducción, dirigido a la aplicación y no dirigido. Para evaluarlos, Vermunt (1994) desarrolló un inventario (Inventory of Learning Styles, ILS) acerca de estrategias de estudio y regulación, motivos para aprender y modelos mentales.

En el primer patrón orientado al significado, el alumno busca comprehender el sentido profundo del material de estudio, establecer relaciones y desarrollar el pensamiento crítico hacia una perspectiva personal. Desde un patrón reproductivo prioriza la memorización y el repaso a fin de aprobar las evaluaciones. En el aplicado, orienta los procesos intelectuales a emplear lo aprendido en situaciones actuales, del mundo real. Finalmente, en el no dirigido experimenta dificultades para procesar el material de estudio, especialmente de la extensión y la complejidad frecuentes en la educación universitaria, y para discriminar entre ideas principales y secundarias (ver Tabla 1).

Tabla 1

Patrones de aprendizaje y sus componentes (Vermunt, 1996, 1998)

\begin{tabular}{|c|c|c|c|c|}
\hline \multirow[b]{2}{*}{ Componentes } & \multicolumn{4}{|c|}{ Patrones de Aprendizaje } \\
\hline & $\begin{array}{l}\text { Dirigido al } \\
\text { significado }\end{array}$ & $\begin{array}{c}\text { Dirigido a la } \\
\text { Aplicación }\end{array}$ & $\begin{array}{l}\text { Dirigido a la } \\
\text { reproducción }\end{array}$ & $\begin{array}{l}\text { No dirigido o } \\
\text { Ambivalente } \\
\end{array}$ \\
\hline $\begin{array}{l}\text { Estrategias de } \\
\text { procesamiento }\end{array}$ & $\begin{array}{l}\text { Profundo, } \\
\text { elaborado y crítico }\end{array}$ & Concreto & $\begin{array}{l}\text { Procesamiento paso a } \\
\text { paso, memorización, } \\
\text { repaso y análisis }\end{array}$ & $\begin{array}{c}\text { Escaso } \\
\text { procesamiento }\end{array}$ \\
\hline $\begin{array}{l}\text { Estrategias de } \\
\text { regulación }\end{array}$ & $\begin{array}{c}\text { Autorregulación de } \\
\text { procesos, } \\
\text { contenidos y } \\
\text { resultados }\end{array}$ & $\begin{array}{l}\text { Ambas: } \\
\text { autorregulación y } \\
\text { regulación externa }\end{array}$ & $\begin{array}{l}\text { Regulación externa de } \\
\text { procesos y resultados }\end{array}$ & $\begin{array}{l}\text { Ausencia de } \\
\text { regulación }\end{array}$ \\
\hline $\begin{array}{l}\text { Concepciones } \\
\text { de aprendizaje }\end{array}$ & $\begin{array}{l}\text { Construcción del } \\
\text { conocimiento }\end{array}$ & Uso del conocimiento & $\begin{array}{l}\text { Incremento del } \\
\text { conocimiento }\end{array}$ & $\begin{array}{l}\text { Estímulo del } \\
\text { docente y } \\
\text { cooperación }\end{array}$ \\
\hline $\begin{array}{l}\text { Orientación del } \\
\text { aprendizaje }\end{array}$ & $\begin{array}{l}\text { Interés personal } \\
\text { como orientación } \\
\text { del aprendizaje }\end{array}$ & $\begin{array}{l}\text { Orientación a la } \\
\text { vocación }\end{array}$ & $\begin{array}{l}\text { Orientación a los } \\
\text { certificados o títulos y } \\
\text { a las calificaciones }\end{array}$ & Ambivalente \\
\hline
\end{tabular}

Fuente: adaptada de Martínez Fernández y García-Ravidá (2012, p. 169) 
Vermunt (1998) postula que el orientado al significado y a la reproducción se relacionan con los estilos homónimos de Entwistle (1998) y con el enfoque "profundo" y "superficial" que distinguen Biggs (1987) y Tait y Entwistle (1996); y que el estilo no orientado exhibe varias similitudes con el que estos últimos autores denominan "estilo apático". También muestra que algunos elementos del orientado a la aplicación refieren al estilo "procesamiento elaborativo" del trabajo de Geisler-Brenstein, Schmeck y Hetherington (1996).

El modelo de Vermunt y su instrumento de diagnóstico se han empleado extensivamente en la investigación en educación superior en países asiáticos (Ajisuksmo y Vermunt, 1999; Marambe, Vermunt Boshuizen, 2012), europeos (Donche y Van Petegem, 2009; Endedijk, Vermunt, Verloop y Brekelmans, 2012; González Gascón, De Juan, Parra Azor, Sarabia Sánchez y Kanther, 2010; Hernández Pina, Rosário, Cuesta Sáez de Tejada, Martínez Clares y Ruiz Lara, 2006; Martínez Fernández y García-Ravidá, 2012) y latinoamericanos (Abalde Paz, Barca Lozano, Muñoz Cantero y Ziemer, 2009; Alves de Lima et al., 2006; de la Barrera, Donolo y Rinaudo, 2010; Vázquez, 2009; González, 2015).

Si bien varios estudios en distintos contextos culturales (entre otros, Busato, Prins, Elshout y Hamaker, 1998; Vázquez, 2009) replican los hallazgos de Vermunt con una correspondencia notable, también la investigación empírica ha mostrado que los cuatro patrones no siempre llegan a conformarse con claridad (Ajisuksmo y Vermunt, 1999; Boyle, Duffy y Dunleavy, 2003; García-Ravidá, de la Barrera, Telleria y Martínez Fernández, en prensa; Martínez Fernández y García-Ravidá, 2012; Marambe, Vermunt y Boshuizen, 2012).

En la mayoría de las investigaciones los patrones de aprendizaje se establecen mediante el análisis factorial de segundo orden (Vermunt, 1998); sin embargo, dado que éste combina variables y no sujetos, resulta complejo para la adscripción individual del patrón predominante. El análisis de conglomerados, por el contrario, ya se ha mostrado conveniente en la investigación previa con el ILS (Vermetten, Vermunt y Lodewijks, 2002; Wierstra y Beerends, 1996) para esta tarea. Los dos últimos autores encuentran tres conglomerados entre estudiantes universitarios que completan el ILS: 1) autorregulado/orientado al significado; 2) externamente regulado/reproductivo; 3) flexible/versátil. El tercero se puede asemejar en cierto modo con el estilo versátil que distinguiera Pask (1976), aquel que se mueve libremente entre estilos según las demandas de la tarea. Vermetten et al. (2002) encuentran dos conglomerados similares (que llaman 
"profundo" y "superficial/no dirigido") y un tercero, que denominan "inactivo", que refiere a los estudiantes que se caracterizan por bajos niveles de actividad de aprendizaje.

Aunque el modelo de Vermunt -como señaláramos- se ha investigado profusamente, hay pocos estudios (Busato et al., 1998; Donche y Van Petegem, 2009; Phan, 2008; Severiens, Ten Dam y Van Hoult Wolters, 2001; Vermetten, Vermunt y Lodewijks, 1999) respecto del desarrollo de los patrones a medida que los alumnos progresan en la carrera. Las investigaciones, por otra parte, muestran resultados disímiles: mientras que algunas evidencian considerables cambios (Donche y Van Petegem, 2009; Phan, 2008), otras no hallan transformaciones significativas en un año y sólo marginales en un período de tres años (Busato et al., 1998; Severiens, Ten Dam y Van Hoult Wolters, 2001).

\section{Desarrollo de los patrones de aprendizaje}

Algunos autores hacen hincapié en que gran parte de las variables personales y contextuales o ambientales, pueden tener un impacto en las concepciones y estrategias de aprendizaje del alumno (Entwistle, McCune y Hounsell, 2003; Entwistle y Ramsden, 1983; Gow y Kember, 1990; Trigwell y Prosser, 1991). El debate respecto de si los estilos de aprendizaje son un "rasgo" o un "estado" es una parte importante de ello. Para algunos investigadores, los enfoques de aprendizaje son, de hecho, características fijas que se relacionan con la personalidad, que son difíciles de modificar en el contexto educativo (Messick, 1996). Otros autores han subrayado su relativa modificabilidad (Entwistle y Ramsden, 1983; Meyer y Muller, 1990; Vermetten et al., 1999; Wierstra y Beerends, 1996).

Vemunt (1992) defiende la postura de que los patrones de aprendizaje exhiben un relativamente alto grado de estabilidad; sin embargo, ello no significa que todos los componentes del aprendizaje deban ser considerados características perdurables. Afirma que algunos componentes de los patrones varían: las estrategias de aprendizaje, en tanto característica de los patrones, son menos estables que las concepciones y las orientaciones del aprendizaje. Vermetten et al. (1999) refieren que las primeras pertenecen al dominio contextual del estudiante, mientras que las segundas al dominio de lo personal. De acuerdo con Vermunt (1992), ello conduce a que las estrategias puedan mostrar una correlación alta con factores del contexto en comparación con las concepciones y orientaciones. En consonancia con este postulado, los escasos estudios longitudinales de los patrones de aprendizaje en la educación superior han indicado que algunos componentes son más susceptibles de cambio que otros. Busato et al. (1998), Vermunt y Minnaert (2003), en sus 
trabajos, señalan que el patrón orientado al significado se incrementa durante la educación superior.

\section{El presente estudio}

En este trabajo deseamos echar luz sobre la cuestión de la modificabilidad de los patrones de aprendizaje durante la permanencia de los estudiantes en la universidad. Se analiza el modelo de Vermunt $(1998,2005)$ en el contexto argentino, a partir de muestras procedentes de dos facultades de Ingeniería con la finalidad de disponer de una base teórica y contextual que nos permita optimizar los procesos de enseñanza y aprendizaje en la universidad.

El tipo de diseño de la investigación es no experimental; se realizan dos estudios: en primer lugar, un estudio transeccional o transversal $(E 1)^{3}$, comparando alumnos de $1^{\circ}$ y de $5^{\circ}$ años; luego, un segundo estudio longitudinal de evolución de grupos (E2) ${ }^{4}$, confrontando dos instancias evaluativas al comienzo y al final del ciclo lectivo en estudiantes de $1^{\circ}$ año.

\subsection{Preguntas de investigación}

Las preguntas que orientan la presente investigación son: 1) ¿Qué patrones de aprendizaje se conforman en los participantes del E1 y E2?, 2) ¿Cómo incide el rendimiento autoinformado y el género en los componentes de los patrones de aprendizaje? y 3) ¿Qué grado de variabilidad evidencian los componentes de los patrones de aprendizaje durante un ciclo lectivo?

En consonancia con los interrogantes planteados, se formulan los siguientes objetivos: 1) Identificar qué patrones de aprendizaje se conforman en E1 y E2, 2) Determinar la incidencia del rendimiento autoinformado y del género en los componentes de los patrones de aprendizaje encontrados en E2, 3) Ponderar la relevancia de las diferencias entre el pretest y el post-test encontradas en el E2.

\section{Metodología}

\subsection{Participantes}

En el estudio transversal (E1) se aplicó el ILS en la Facultad de Ingeniería, Universidad Nacional de Cuyo (Mendoza, Argentina), a 149 alumnos de 1ำ año, 119 hombres (80\%) y 30

\footnotetext{
${ }^{3}$ De aquí en más el estudio transversal -primer estudio- se identificará con la sigla E1.
}

${ }^{4}$ El estudio longitudinal -segundo estudio-, con la sigla E2. 
mujeres (20\%), con una edad comprendida entre 17 y 40 años $(M=18,67 ; D E=2,13)$ y a 90 alumnos, 77 hombres (87\%) y 12 mujeres (13\%) de 5ำ, con una edad comprendida entre 20 y 35 años $(M=24,64 ; D E=2,62)$. En el estudio longitudinal (E2), a 110 alumnos, 40 varones (36\%) y 70 mujeres (64\%), con una edad comprendida entre 17 y 42 años $(M=19,38 ; D E=$ $4,0)$ de $1^{\circ}$ año de la Facultad de Ingeniería en Ciencias Agrarias, Universidad Nacional de Cuyo. En cuanto al tipo de muestra, se selecciona de manera intencional; en este caso, los primeros y últimos años de las carreras de Ingeniería en estudio.

Dado que en esta investigación se trabaja con una muestra de estudiantes de la Universidad Nacional de Cuyo de dos facultades diferentes, es necesario señalar que ambas se caracterizan por ofrecer carreras de corte científico-técnico (la Facultad de Ingeniería a través de las siguientes carreras: Ingeniería en Petróleo, Civil, Industrial y Mecatrónica; la Facultad de Ciencias agrarias: Ingeniería en Ciencias Agrarias, en Recursos Naturales Renovables, Licenciatura en Bromatología y en Enología). Esto es, si bien los estudiantes provienen de diferentes carreras ingenieriles o técnicas, sus perfiles son semejantes, permitiendo el análisis conjunto en el presente estudio.

\subsection{Procedimiento}

Los instrumentos fueron aplicados en cada clase con la asistencia del profesor, quien invitó de manera voluntaria a los estudiantes a participar y les anticipó que, a aquellos que no desearan responder, les propondría otra actividad; pero, no fue necesario hacerlo porque todos prefirieron completar los instrumentos, elección que asimilamos a un consentimiento informado. Se les comunicaron, entonces, los objetivos del estudio, se les indicó que podían interrumpir su participación en cualquier momento del trabajo (no obstante, todos lo finalizaron) y se les señaló que los inventarios podían ser completados consignando el nombre o de manera anónima. Se respetó el principio de confidencialidad y anonimato, ya que la única persona que tuvo acceso a los datos fue la primera investigadora, y luego en el análisis desestimamos los nombres de los respondientes. Del total de alumnos en cada estudio -en el transversal (E1), 149 en $1^{\circ}$ año y 90 en 5ํaño; en el longitudinal (E2), 110-, la muestra quedó constituida con el $100 \%$ de los sujetos. Respecto de la edad, en la institución universitaria de la que provienen las muestras, el alumno desde primer año es considerado el responsable último de su trayectoria académica, por lo cual no se solicita el consentimiento a los padres para ninguna actividad docente ni investigativa. 


\subsection{Instrumento}

En primer lugar, los participantes completaron una ficha de datos demográficos donde se indaga acerca de ciertos factores personales y contextuales. Entre ellos, se pregunta sobre edad, sexo, esfuerzo, nota media, percepción sobre el rendimiento previo y si realizan otras actividades complementarias al estudio.

Seguidamente, se aplicó el ILS -Inventory of Learning Styles- de Jan Vermunt (1998) en la versión en español, traducida y validada en $2009^{5}$.

El ILS se compone de 120 afirmaciones que abarcan los cuatro componentes del aprendizaje definidos por Vermunt. Para los ítems sobre estrategias (de procesamiento y de regulación), los estudiantes deben indicar en una escala de cinco puntos en qué grado prefieren utilizar, durante el estudio, las actividades referidas. Dicha escala varía de (1) "lo hago rara vez o nunca" a (5) "lo hago siempre". Para los ítems relativos a las orientaciones y concepciones de aprendizaje, se les solicita que señalen en una escala de cinco puntos el grado en que los puntos de vista y los motivos descritos se corresponden con sus propios puntos de vista y motivos. Aquí la escala varía de (1) "totalmente en desacuerdo" a (5) "completamente de acuerdo". Cabe señalar que el ILS genera cuatro escalas, cada una con cinco subescalas, dando un total de veinte ${ }^{6}$.

\section{Análisis de los datos}

En E1, se identifican los patrones de aprendizaje que se conforman y se exploran las diferencias entre los alumnos de $1^{\circ}$ y de $5^{\circ}$. Para ello, se efectúa en primer lugar un análisis jerárquico de conglomerados (método WARD) para identificar grupos relativamente homogéneos respecto de las características de aprendizaje. Las diferencias entre conglomerados se estudian en su significación por medio del análisis de varianza. La

\footnotetext{
${ }^{5} \mathrm{El}$ instrumento ha sido traducido al español por miembros de un equipo internacional denominado G_PAFIU, Grupo de Investigación, docencia e intervención en Patrones de Aprendizaje y Formación Investigadora en la Universidad, liderado por J. Reinaldo Martínez Fernández desde la Universidad Autónoma de Barcelona, del cual forman parte las autoras del presente artículo desde 2011.

${ }^{6}$ A continuación, se presenta cada una de las escalas y subescalas y se incluye el puntaje total posible en ellas. La escala estrategias de procesamiento contiene las siguientes dimensiones: 1) relacionar y estructurar, cuyo rango teórico es de 7 a 35; 2) procesamiento crítico, 4 a 20; 3) memorizar y repetir, de 5 a 25; 4 ) analizar, 6 a 30; y 5) procesamiento concreto, de 5 a 20. La escala estrategias de regulación, las siguientes: 1) autorregulación de procesos, 7 a 35; 2) autorregulación de contenidos, 4 a 20; 3) regulación externa de procesos, 6 a 30; 4) regulación externa de resultados, 5 a 25; y 5) falta de regulación, 6 a 30. La escala modelos mentales se conforma de: 1) construcción del conocimiento, 9 a 45; 2) incorporación del conocimiento, 9 a 45; 3) uso del conocimiento, 6 a 30;4) conocimiento como estímulo, 8 a 40; y 5) conocimiento por cooperación, 8 a 40 . Por último, la escala orientaciones del aprendizaje presenta las siguientes dimensiones: 1) orientación al interés personal, 2) orientación a la acreditación, 3) orientación a la autoevaluación, 4) orientación a la profesión y 5) no orientado; todas presentan el mismo rango teórico de 5 a 25.
} 
relación entre año de estudio y patrones se analiza desde la prueba de $\mathrm{Chi}^{2}$. En el E2, se examinan los cambios intra-individuales en las medias de las escalas entre la primera y la segunda aplicación del ILS, con la prueba t y el cálculo del tamaño del efecto. Luego, se contrastan los patrones hallados a través de un análisis jerárquico de conglomerados en las dos instancias evaluativas. Finalmente, se analizan las diferencias en función de las variables personales en estudio (rendimiento autoinformado y género) en los dominios que conforman los patrones de aprendizaje mediante el análisis de varianza de un factor y la prueba t. Todos los análisis de ambos estudios se realizaron con el programa SPSS.

Se efectuaron los análisis de confiabilidad de las escalas del ILS, la tabla 2 muestra el alpha de Cronbach para E1 y E2, el cual oscila entre 0,29 y 0,17, respectivamente -los índices más bajos, correspondientes a interés personal- y 0,83 -el más alto en $\mathrm{E} 1$, que alcanza conocimiento como cooperación- y 0,89 -el más alto en E2, para conocimiento por estímulo-.

Tabla 2

Análisis de confiabilidad de las escalas del ILS para E1 y E2

\begin{tabular}{lllll}
\hline Escalas & E1 & Alpha & & \\
& & E2 & $N^{\circ}$ de ítems \\
\hline
\end{tabular}

\section{Estrategias de procesamiento}

Relacionar y estructurar

$\begin{array}{lll}\mathbf{0 , 7 6} & \mathbf{0 , 7 3} & 7 \\ 0,62 & 0,42 & 4 \\ 0,77 & \mathbf{0 , 8 0} & 5 \\ 0,66 & 0,70 & 6 \\ 0,67 & 0,62 & 5\end{array}$

Estrategias de regulación

Autorregulación de procesos

Autorregulación de contenidos

$\begin{array}{lll}0,62 & 0,70 & 7 \\ \mathbf{0 , 6 9} & \mathbf{0 , 7 2} & 4 \\ 0,45 & 0,48 & 6 \\ 0,64 & 0,48 & 5 \\ 0,58 & 0,71 & 6\end{array}$

\section{Modelos mentales}

Construcción del conocimiento

$\begin{array}{lll}0,66 & 0,75 & 9 \\ 0,68 & 0,75 & 9 \\ \mathbf{0 , 7 0} & 0,62 & 6 \\ \mathbf{0 , 8 2} & \mathbf{0 , 8 9} & 8 \\ \mathbf{0 , 8 3} & \mathbf{0 , 7 2} & \mathbf{8}\end{array}$




\begin{tabular}{llll} 
Orientaciones de aprendizaje & & & \\
Interés personal & 0,297 & 0,17 & 5 \\
Acreditación & 0,53 & 0,58 & 5 \\
Autoevaluación & 0,79 & 0,57 & 5 \\
Profesión & 0,67 & $\mathbf{0 , 7 1}$ & 5 \\
No orientado & 0,69 & $\mathbf{0 , 7 3}$ & 5 \\
\hline
\end{tabular}

\section{Resultados}

\subsection{Estudio 1}

En una primera etapa, se implementó un análisis jerárquico de conglomerados. El análisis con las 20 subescalas del ILS mostró que la mejor solución es en cuatro grupos. EI primer conglomerado está conformado por estrategias de procesamiento profundo (relación y estructuración, pensamiento crítico), análisis y pensamiento concreto, estrategias de autorregulación de procesos, resultados y contenidos del aprendizaje y orientación personal. En consecuencia, corresponde muy cercanamente al patrón dirigido al significado (Vermunt, 1998). En el segundo conglomerado, se incluye la memorización y regulación externa de procesos y de resultados, la orientación a la acreditación, a la autoevaluación y también conocimiento como incorporación. Se trata, entonces, del patrón de aprendizaje dirigido a la reproducción (Vermunt, 1998, 2005). En el tercero, las restantes concepciones del aprendizaje y la orientación a la profesión, lo cual podría constituir un patrón versátil. El cuarto conglomerado se caracteriza por falta de regulación y de orientación (u orientación ambivalente) conformando un patrón no orientado.

Luego, evaluamos si ciertos patrones aparecen con mayor o menor frecuencia en $1^{\circ} \mathrm{y}$ en $5^{\circ}$ años (ver Tabla 3). La prueba de $\mathrm{Chi}^{2}$ indica que, en comparación con los alumnos de $5^{\circ}$, en $1^{\circ}$ año hay más alumnos reproductivos/no dirigidos y menos orientados al significado y del patrón versátil $(p<0,001)$.

\footnotetext{
7 Se incrementa a 0,37 si se desestima el ítem № 69: "Considero estos estudios como simple relajación". Este resultado replica la comprobación de Stella Vázquez (2009, p. 116): "En la escala de orientación al interés personal se destaca el ítem referido al interés por logros de alto nivel en el estudio, que en la escala original de Vermunt integra la escala de orientación a la acreditación. El cambio tiene sentido desde la teoría y parece indicar que la referencia al nivel de logro se comprende mejor dentro de la motivación intrínseca (...)".
} 
Tabla 3

Descripción de patrones de aprendizaje según curso E1 ( $N=238)$

\begin{tabular}{|c|c|c|c|c|c|c|}
\hline & & \multicolumn{4}{|c|}{ Patrón } & \multirow[t]{2}{*}{ Total } \\
\hline \multirow{3}{*}{ CURSO } & & Significado & Reproductivo & Versátil & $\begin{array}{c}\text { No } \\
\text { orientado }\end{array}$ & \\
\hline & $1^{\circ}$ & $32(21,5 \%)$ & $51(34,3 \%)$ & $33(22,1 \%)$ & $33(22,1 \%)$ & 149 \\
\hline & $5^{\circ}$ & $33(37 \%)$ & $5(6 \%)$ & $33(37 \%)$ & $18(20 \%)$ & 89 \\
\hline \multicolumn{2}{|c|}{ Total } & $65(27,3 \%)$ & $56(23,5 \%)$ & $66(27,7 \%)$ & $51(21,5 \%)$ & 238 \\
\hline
\end{tabular}

\subsection{Estudio 2}

En primer lugar, examinamos si se verifican diferencias significativas en las medias de las puntuaciones de las 20 subescalas del ILS entre el pre-test y el post test mediante la prueba t; los resultados se presentan en la Tabla 4 (ver Tabla 4). En segundo lugar, calculamos el tamaño del efecto (d de Cohen), el que oscila alrededor de 0,10, esto es, un efecto pequeño.

Tabla 4

Resultados de las subescalas del ILS para pre-test y pos-tes. Prueba $t, d$ E2 ( $N=\mathbf{2 0 8 )}$

\begin{tabular}{|c|c|c|c|c|c|c|}
\hline \multirow[b]{2}{*}{ Escalas } & \multicolumn{2}{|c|}{ Pre - test $N=110$} & \multicolumn{2}{|c|}{ Pos -test $\mathrm{N}=98$} & \multirow[t]{2}{*}{$\mathrm{t}(208)$} & \multirow[t]{2}{*}{$D$} \\
\hline & $M$ & $D S$ & $M$ & DS & & \\
\hline \multicolumn{7}{|l|}{ Estrategias de procesamiento } \\
\hline Relacionar y estructurar & 3,20 & 0,67 & 3,53 & 0,61 & $-3,753$ & 0,11 \\
\hline Procesamiento crítico & 3,06 & 1,29 & 3,20 & 0,73 & $-0,996$ & \\
\hline Memorizar y repetir & 2,96 & 0,96 & 3,12 & 0,88 & $-1,248$ & \\
\hline Analizar & 3,42 & 0,63 & 3,59 & 0,97 & $-1,827$ & \\
\hline Procesamiento concreto & 3,59 & 0,62 & 3,77 & 0,61 & $-2,125$ & 0,10 \\
\hline \multicolumn{7}{|l|}{ Estrategias de regulación } \\
\hline Autorregulación de procesos & 3,37 & 0,68 & 3,55 & 0,66 & $-1,938$ & 0,11 \\
\hline Autorregulación de contenidos & 2,76 & 0,80 & 3,03 & 0,83 & $-2,341$ & 0,13 \\
\hline Regulación externa de procesos & 3,22 & 0,58 & 3,34 & 0,54 & $-1,627$ & \\
\hline Regulación externa de resultados & 3,60 & 0,54 & 3,75 & 0,55 & $-1,973$ & \\
\hline Falta de regulación & 3,51 & 0,89 & 3,60 & 0,90 & $-0,668$ & \\
\hline \multicolumn{7}{|l|}{ Orientaciones de aprendizaje } \\
\hline Interés personal & 3,36 & 0,54 & 3,43 & 0,46 & $-0,688$ & \\
\hline Acreditación & 3,42 & 0,76 & 3,44 & 0,70 & $-0,204$ & \\
\hline Autoevaluación & 3,97 & 0,78 & 3,89 & 0,78 & 0,725 & \\
\hline Profesión & 4,34 & 0,63 & 4,21 & 0,71 & 1,372 & \\
\hline No orientado & 2,42 & 0,80 & 2,52 & 0,95 & $-0,823$ & \\
\hline
\end{tabular}




\begin{tabular}{llllll} 
Modelos mentales & & & & & \\
Construcción del conocimiento & 4,13 & 0,46 & 4,07 & 0,52 & 0,896 \\
Incorporación del conocimiento & 3,82 & 0,55 & 3,67 & 0,65 & 1,774 \\
Uso del conocimiento & 4,37 & 0,40 & 4,25 & 0,52 & 1,762 \\
Conocimiento por estímulo & 3,70 & 0,69 & 3,64 & 0,88 & 0,540 \\
Conocimiento por cooperación & 3,79 & 0,60 & 3,74 & 0,67 & 0,541 \\
\hline
\end{tabular}

En general, los alumnos de $1^{\circ}$ año en el post-test, en comparación con la instancia inicial, conceptualizan el aprendizaje menos como estímulo $(M=3,64, M=3,70 ; D E=0,88$, $D E=0,69)$, evidencian un incremento de las actividades de autorregulación de procesos $(M=$ $3,55, M=3,37 ; D E=0,68, D E=0,66)$ y de autorregulación de contenidos $(M=3,03, M=2,76$; $D E=0,83, D E=0,80$ ), al tiempo que muestran un decrecimiento de la orientación a la autoevaluación $(M=3,89, M=3,93 ; D E=0,78, D E=0,78)$. No encontramos diferencias significativas en las puntuaciones medias respecto de la regulación externa ( $M=3,75, M=$ 3,60; $D E=0,55, D E=0,54)$ y de las estrategias de análisis $(M=3,59, M=3,42 ; D E=0,97, D E=$ $0,63)$.

En segundo lugar, se realizó un análisis jerárquico de conglomerados, en el cual nuevamente se conformaron cuatro grupos (ver Tabla 5).

Tabla 5

Descripción de patrones de aprendizaje según instancia de aplicación E2 ( $N=208)$

\begin{tabular}{|c|c|c|c|c|c|c|}
\hline & & \multicolumn{4}{|c|}{ Patrón } & \multirow[t]{2}{*}{ Total } \\
\hline & & Significado & Reproductivo & Versátil & $\begin{array}{c}\text { No } \\
\text { orientado }\end{array}$ & \\
\hline \multirow[t]{2}{*}{ INSTANCIAS } & PRE 1 & $14(13 \%)$ & $31(28 \%)$ & $31(28 \%)$ & $34(31 \%)$ & 110 \\
\hline & POST 2 & $28(29 \%)$ & $20(20 \%)$ & $25(25.5 \%)$ & $25(25.5 \%)$ & 98 \\
\hline Total & & $42(20 \%)$ & $51(27 \%$ & $56(29 \%)$ & $59(29 \%)$ & 208 \\
\hline
\end{tabular}

El primero corresponde al patrón orientado al significado, el segundo al reproductivo, el tercero al versátil y el cuarto es el no orientado. Se observa que aumenta el porcentaje de alumnos orientados al significado y decrece el porcentaje de los alumnos orientados a la reproducción en la instancia pos-test, como así también los correspondientes a los patrones versátil y no orientado. 
Por último, analizamos la relación de las subescalas del ILS con el rendimiento autoinformado $^{8}$ y el género. El primero se categorizó en tres: bajo $(M=5,25 ; D E=1,3)$, medio $(M=7,06 ; D E=1,8)$ y alto $(M=9,03 ; D E=2,1)(F=3,489 ; p<0,05)$. Se hallan diferencias significativas para la instancia final pos-test en los siguientes componentes: falta de regulación, bajo $(M=19,1 ; D E=4,6)$, medio $(M=18,2 ; D E=3,1)$, alto $(M=14,4 ; D E=4,1)(F=$ 8,588; $p<0,01)$, orientación a la acreditación, bajo $(M=15,9 ; D E=3,6)$, medio $(M=17,9 ; D E=$ $3,4)$, alto $(M=16,7 ; D E=3,3)(F=2,991 ; p=0,05)$, conocimiento como uso, bajo $(M=23,4$; $D E=3,2)$, medio $(M=25,7 ; D E=3,3)$, alto $(M=26,8 ; D E=2,3)(F=3,567 ; p<0,05)$.

En cuanto al género, se evidencia que en ambas instancias evaluativas las mujeres presentan puntuaciones más altas respecto de los varones en conocimiento como construcción, tanto en el pre-test $(M=37,3 ; D E=4,7$ vs. $M=37,2 ; D E=4,3)(t=2,67 ; p<0,05)$ como en el pos-test $(M=35,1 ; D E=4,3$ vs. $M=37,2 ; D=4,3)(t=-2,14 ; p<0,04)$.

Finalmente, es importante destacar el empleo de estudios longitudinales ${ }^{9}$ y transversales ${ }^{10}$, ya que los primeros permiten captar las diferencias intraindividuales y no se hallan problemas de homogeneidad entre grupos. En los segundos se ofrece información general respecto a las diferencias de grupos en diferentes momentos de análisis.

\section{Discusión}

En este estudio con alumnos universitarios de Ingeniería se intentó dar respuesta a los objetivos que guiaron el análisis de los resultados. En cuanto al primero -Identificar qué patrones de aprendizaje se conforman en E1 y E2-, en el estudio transversal (E1) los patrones no se conforman de manera idéntica a los hallados por Vermunt $(1998,2005)$. Sin embargo, se distingue un patrón orientado al significado y uno reproductivo. El primero se incrementa con el tiempo y el segundo, decrece, mostrando una relación sistemática entre curso y patrón; específicamente, el patrón orientado al significado es característico del último año y el reproductivo del primero. Ello nos permite afirmar que se hallan diferencias significativas debidas al desarrollo de los patrones durante la permanencia en la universidad

\footnotetext{
8 Rendimiento autoinformado refiere a la percepción que tiene el estudiante de su propio desempeño y/o rendimiento académico, este aspecto es contestado por el alumno en uno de los ítems del ILS, que se enuncia de la siguiente manera: ¿Cómo valorarías tu esfuerzo o dedicación al aprendizaje en la escuela secundaria? Puntuación de 0 a 10. Se espera que este ítem tenga incidencia en los patrones de aprendizaje esto se debe a qué un estudiante con una percepción baja de su rendimiento podría corresponder con un patrón no orientado o ambivalente, a diferencia de un estudiante con una percepción alta de su rendimiento debería ser coherente con un patrón orientado al significado.

${ }^{9}$ Consisten en observar a un mismo grupo de sujetos a lo largo de un período de tiempo.

10 Consisten en estudiar en un momento determinado a distintos grupos de sujetos.
} 
a favor del patrón profundo, lo que es coherente con la autorregulación del aprendizaje, que se va gestando a medida que se avanza en la carrera universitaria.

En cuanto al estudio longitudinal (E2), se contrastaron los patrones hallados a través de un análisis jerárquico de conglomerados en las dos instancias evaluativas, que mostró que la mejor solución es en cuatro grupos: tres correspondientes a los patrones del modelo de Vermunt (dirigido al significado, dirigido a la reproducción y no orientado) y un cuarto patrón "versátil", similar al que identifican Wierstra y Beerends (1996). Por otra parte, los alumnos en el post-test demuestran mayor desarrollo de las estrategias de autorregulación de contenidos y de resultados.

Con respecto al segundo objetivo -Determinar la incidencia del rendimiento autoinformado y del género en los componentes de los patrones de aprendizaje encontrados en E2-, se hallan relaciones significativas entre niveles de rendimiento autoinformado y empleo diferencial de las actividades de estudio: al nivel alto de desempeño corresponde el manejo frecuente de estrategias profundas y de autorregulación. La falta de regulación se corresponde con aquellos estudiantes que manifiestan un rendimiento bajo y decrece en aquellos de rendimiento alto. Según el género, las diferencias se hallan a favor de las mujeres en el conocimiento como construcción.

En relación con el tercer objetivo -Ponderar la relevancia de las diferencias entre el pretest y el post-test encontradas en el E2-, se analizaron los cambios intra-individuales en las medias de las escalas entre la primera y la segunda aplicación del ILS, a través de la prueba t y el cálculo del tamaño del efecto, el que oscila alrededor de 0,10 , lo cual representa un efecto pequeño. Se evidenciaron avances notables en la instancia post-test en las subescalas de relacionar y estructurar, procesamiento concreto, estrategias de regulación tanto de procesos como de contenidos.

En consonancia con los resultados, podemos decir que: en cuanto al estudio transversal: a) se logra la modificabilidad de los patrones de aprendizaje y esto se evidencia a medida que el estudiante avanza en su carrera universitaria, b) no se conforman de manera idéntica los patrones hallados por Vermunt, pero se encuentran los polos opuestos del modelo -un patrón orientado al significado y uno reproductivo- y en cuanto al estudio longitudinal, a) se corrobora que en la medida que el estudiante avanza desarrolla mayores estrategias de autorregulación en el aprendizaje, esto se evidencia en los alumnos que realizan el post-test que demuestran mayor desarrollo en las estrategias, b) los patrones que 
se conforman según el modelo de Vermunt son dirigido al significado, dirigido a la reproducción y no orientado.

Finalmente, es necesario destacar la importancia de la modificabilidad de los patrones de aprendizaje, así, para Vermunt, los patrones tienen el potencial de desarrollarse a través del tiempo, y es un constructo dependiente de diferentes contextos y de ciertas características personales, lo que permitiría configurar una preferencia del estudiante por formas específicas para acceder, procesar, regular, producir y orientar motivacionalmente sus acciones de aprendizaje (Martínez -Fernández y García Ravidá, 2012).

En suma, este estudio señala la necesidad de continuar con una línea de investigación que se plantea a futuro: la necesidad de ponderar en qué grado los factores personales (género, valor del esfuerzo, tiempo dedicado al estudio -variables que también evalúa el ILS) y contextuales (por ej.: naturaleza del currículum) se asocian a la consistencia y variabilidad de los patrones a través del tiempo. La evaluación de los segundos exigiría complementar el ILS con otro/s instrumento/s.

\section{Referencias}

Abalde Paz, Eduardo, Barca Lozano, Alfonso, Muñoz Cantero, Jesús Miguel y Ziemer, Marcos Fernando. (2009). Rendimiento académico y enfoques de aprendizaje: una aproximación a la realidad de la enseñanza superior brasileña en la región norte. Revista de Investigación Educativa, 27(2), 303-319.

Aguilar Rivera, María del Carmen (2010) Los enfoques de aprendizaje en la universidad: un estudio de caso. Revista de Psicología, 6 (11), 67-86.

Ajisuksmo, Clara y Vermunt, Jan. (1999). Learning styles and self-regulation of learning at university: An Indonesian study. Asia Pacific Journal of Education, 19(2), 45-59.

Alonso Tapia, Jesús. (1997). Motivar para el aprendizaje. Teorías y estrategias. Barcelona: EDEBE.

Alves de Lima, Alberto, Betatti, María Inés, Baratta, Sergio, Falconi, Mariano, Sokn, Fernando, Galli, Amanda, Barrero, Carlos, Cagide, Arturo e Iglesias, Ricardo. (2006). Learning Strategies used by Cardiology Residents: Assessment of Learning Styles and their correlations. Education for Health, 19(3), 289-297.

Bernard, Juan Antonio. (1993). Estrategias de aprendizaje-enseñanza: evaluación de una actividad compartida en la escuela. En Carles Monereo (Comp.), Las estrategias de aprendizaje. Procesos, contenidos e interacción (pp. 15-29). Barcelona: Domenech.

Biggs, John. (1987). Student approaches to learning and studying. Hawthorn: Australian Council for Educational Research. 
Boyle, Elizabeth, Duffy, Tim y Dunleavy, Karen. (2003). Learning styles and academic outcome: The validity and utility of Vermunt's Inventory of Learning Styles in a British higher education setting. British Journal of Educational Psychology, 73, 267-290.

Busato, Vittorio, Prins, Frans, Elshout, Jan y Hamaker, Christiaan. (1998). Learning styles: A cross-sectional and longitudinal study in higher education. British Journal of Educational Psychology, 68, 427-441.

de la Barrera, María Laura, Donolo, Danilo y Rinaudo, María Cristina. (2010). Estilos de aprendizaje de los alumnos: peculiaridades al momento de aprender. Revista Estilos de Aprendizaje, 6(6), 1-25.

Difabio de Anglat, Hilda. (1999). Metacognición y aprendizaje significativo en el nivel universitario. Psico/Pedagógica, 4, 11-27.

Donche, Vincent y Van Petegem, Peter. (2009). The development of learning patterns of student teachers: a cross-sectional and Iongitudinal study. Higher Education, 57, 463475.

Endedijk, Maaike, Vermunt, Jan, Verloop, Nico y Brekelmans, Mieke. (2012). The nature of student teachers' regulation of learning in teacher education. British Journal of Educational Psychology, 82(3), 469-491. doi: 10.1111/j.2044-8279.2011.02040.x

Entwistle, Noel (1998). Improving teaching through research on student learning. In J. J. Forest (Ed.). University teaching: International perspectives (pp. 72-112). New York: Garland.

Entwistle, Noel y Ramsden, Paul. (1983). Understanding student learning. London: Croom Helm.

Entwistle, Noel, McCune, Velda y Hounsell, Jenny. (2003). Investigating ways of enhancing university teaching-learning environments: Measuring students' approaches to studying and perceptions of teaching. In Erik De Corte, Lieven Verschaffel, Noël Entwistle, \& Jeroen van Merrienboer (Eds.). Powerful learning environments: unravelling basic components and dimensions, advances in learning and instruction series (pp. 89-107). London: Pergamon.

Entwistle, Noël, McCune, Velda y Walker, Matthew. (2001). Conceptions, styles, and approaches within higher education: analytical abstractions and everyday experience. Perspectives on cognitive, learning and thinking styles. Lawrence Erlbaum Associates: New Jersey.

Flavell, John H. (1987). Speculations about the nature and development of metacognition. In Franz E. Weinert, y Rainer H. Kluwe (Eds.), Metacognition, motivation and understanding (pp. 21-29). Hillsdale, NJ: Erlbaum.

García Retana, José Ángel. (2013). Reflexiones sobre los estilos de aprendizaje y el aprendizaje del cálculo para Ingeniería. Revista Electrónica Actualidades Investigativas en Educación, 13(1), 1-28. Recuperado de http://revista.inie.ucr.ac.cr/index.php/aie/article/view/612 
García-Fuentes, Carmen Delia, Muñoz Cantero, J. Miguel y Abalde Paz, Eduardo. (2002). Universitarios y profesionales, diagnóstico de estilos de aprendizaje. Revista de investigación educativa, 20(2), 339- 356.

García-Ravidá, Laura, de la Barrera, María Laura, Telleria, María Begoña, y MartínezFernández, José Reinaldo (en prensa). Patrones de aprendizaje en la universidad: variables personales y contextuales relacionadas en una muestra de estudiantes venezolanos de educación. Anales de Psicología.

Geisler-Brenstein, E., Schmeck, R. y Hetherington, J. (1996). An individual difference perspective on student diversity. Higher Education, 31(1), 73-96.

González Gascón, Elena, De Juan, María D., Parra Azor, José F., Sarabia Sánchez, Francisco J. y Kanther, Andreas. (2010). Aprendizaje autorregulado: antecedentes y aplicación a la docencia universitaria de marketing. Revista de Investigación Educativa, 28(1), 171-194.

González, Mariela. (2015). Hacia un modelo multidimensional de la motivación orientada al futuro en relación con el aprendizaje autorregulado. Estudio empírico y ciclos de diseño en estudiantes de Ingeniería Agronómica y Ciencias de la Educación. (Tesis doctoral inédita). Universidad Nacional de Cuyo, Facultad de Filosofía y Letras, Mendoza, Argentina.

Gow, Lyn y Kember, David. (1990). Does higher education promote independent learning? Higher Education, 19(3), 307-322.

Hernández Pina, Fuensante, Rosário, Pedro, Cuesta Sáez de Tejada, José David, Martínez Clares, Pilar y Ruiz Lara, Encarna. (2006). Promoción del aprendizaje estratégico y competencias de aprendizajeen estudiantes de primero de Universidad: evaluación de una intervención. Revista de Investigación Educativa, 24(2), 615-631.

Jonassen, David and Grabowski, Bárbara. (1993). Handbook of individual differences learning and instruction. Lawrence Erlbaum associates, publishers. New Jersey: London.

Kolb, David A. (1984). Experiential Learning. Englewood Cliffs, NJ: Prentice-Hall.

Marambe, Kosala N., Vermunt, Jan D. y Boshuizen, Henny P.A. (2012). A cross-cultural comparison of student learning patterns in higher education. Higher Education, 64(3), 299-316. doi: 10.1007/s10734-011-9494-z.

Marín Gracia, María Ángeles. (2002). La investigación sobre diagnóstico de los estilos de aprendizaje en la enseñanza superior. Revista de Investigación Educativa - RIE, 20(2), 303-337.

Martínez Fernández, José Reinaldo y García-Ravidá, Laura. (2012). Patrones de aprendizaje en estudiantes universitarios del Máster en Educación Secundaria: variables personales y contextuales relacionadas. Revista de Currículum y Formación del Profesorado, 16(1), 166-182. 
Martínez Fernández, José Reinaldo, Villegas, María Elena y Martínez Torres, Mercedes. (2004). Concepciones de aprendizaje y estrategias metacognitivas en universitarios venezolanos y españoles. Revista Latina de Pensamiento y Lenguaje, 12(1), 21-35.

Marton, Ference y Säljö, Roger. (1976). On qualitative differences in learning - I: Outcome and process. British Journal of Educational Psychology, 46, 4-11.

Messick, Samuel. (1996). Cognitive styles and learning. In Eric De Corte y Franz Weinert (Eds.), International encyclopaedia of developmental and instructional psychology (pp. 638-641). Oxford: Elsevier Science.

Nuttin, Joseph. (1980). Motivation et perspective d'avenir. Lovaina, Bélgica: Presses Universitaires.

Nuttin, Joseph. (1982). Teoría de la motivación humana. De la necesidad al proyecto de acción. Barcelona: Paidós.

Pask, Gordon. (1976). Styles and strategies of learning. British Journal of Educational Psychology, 49, 128-148.

Phan, Huy P. (2008). Predicting change in epistemological beliefs, reflective thinking and learning styles: A longitudinal study. British Journal of Educational Psychology, 78, 7593.

Pintrich, Paul y García, Teresa. (1993). Intraindividual differences in students' motivation and self-regulated learning. German Journal of Educational Psychology, 7(3), 99-107.

Sadler-Smith, Eugene. (1996). Approaches to studying: Age, gender and academic performance. Educational Studies, 22(3), 367-379. doi:10.1080/0305569960220306.

Säljö, Roger. (1979). Learning about learning. Higher Education, 8, 443-451. doi:10.1007/BF01680533.

Severiens, Sabine, Ten Dam, Geert y Van Hoult Wolters, Bernardette. (2001). Stability of processing and regulation strategies: Two longitudinal studies on student learning. Higher Education, 42, 437-453.

Tait, Hillary y Entwistle, Noel. (1996). Identifying students at risk through ineffective study strategies. Higher Education, 31, 97-116. doi:10.1007/BF00129109.

Trigwell, Keith, y Prosser, Michael. (1991). Improving the quality of student learning: the influence of learning context and student approaches to learning on learning outcomes. Higher Education, 22(3), 251-266.

Vázquez, Stella Maris. (2009). Rendimiento académico y patrones de aprendizaje en estudiantes de Ingeniería. Ingeniería y Universidad, 13(1), 105-136.

Vermetten, Yvonne, Vermunt, Jan y Lodewijks, Hans. (1999). A longitudinal perspective on learning strategies in higher education - different view-points towards development. British Journal of Educational Psychology, 69(2), 221-242. 
Vermetten, Yvonne, Vermunt, Jan y Lodewijks, Hans. (2002). Powerful learning environments? How do students differ in their response to instructional measures. Learning \& Instruction, 12, 263-284.

Vermunt, Jan y Minnaert, Alexander. (2003). Dissonance in student learning patterns: When to revise theory? Studies in Higher Education, 28(1), 49-61. doi:10.1080/03075070309301.

Vermunt, Jan. (1992). Leerstijlen en sturen van leerprocessen in het hoger onderwijs: Naar procesgerichte instructie in zelfstandig denken [Learning styles and regulation of learning in higher education: Toward process-oriented instruction in autonomous thinking]. Amsterdam/Lisse: Swets \& Zeitlinger.

Vermunt, Jan. (1994). Inventory of learning styles in higher education: scoring key. Tilburg University: Department of Educational Psychology.

Vermunt, Jan. (1996). Metacognitive, cognitive and affective aspects of learning styles and strategies: a phenomenographic analysis. Higher Education, 31, 25-50.

Vermunt, Jan. (1998). The regulation of constructive learning processes. British Journal of Educational Psychology, 68, 149-171.

Vermunt, Jan. (2005). Relations between student learning patterns and personal and contextual factors and academic performance. Higher Education, 49(3), 205-234. doi:10.1007/s10734-004-6664-2.

Wierstra, Ronny y Beerends, Eva (1996). Leeromgeving en leerstrategieen van eerstejaarsstidenten sociale wetenschappen. Tijdschrift voor Onderwijsrsearch, 21(4), 300-322.

Wittrock, Merlin C. (1991). Testing and recent research in cognition. In M. C. Wittrock, \& E. L. Baker (Eds.). Testing and cognition (pp. 5-16). Englewood Cliffs, NJ: Prentice-Hall.

Zimmerman, Barry J. (2000). Attaining self-regulation: a social cognitive perspective. In Monique Boekaerts, Paul Pintrich, y Moshe Zeidner (Eds), Handbook of SelfRegulation (pp. 13-38). San Diego: Academic Press. 